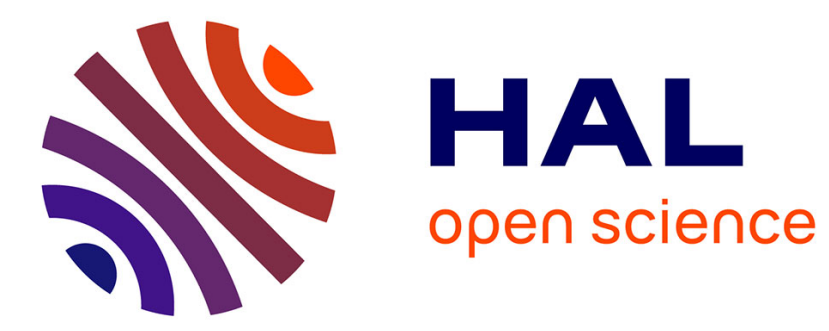

\title{
Diagnosis of an uncertain static system
}

Olivier Adrot, Didier Maquin, José Ragot

\section{To cite this version:}

Olivier Adrot, Didier Maquin, José Ragot. Diagnosis of an uncertain static system. 39th IEEE Conference on Decision and Control, CDC 2000, Dec 2000, Darling Harbour, Australia. pp.4150-4154, 10.1109/CDC.2000.912366 . hal-00201416

\section{HAL Id: hal-00201416 https://hal.science/hal-00201416}

Submitted on 31 Mar 2014

HAL is a multi-disciplinary open access archive for the deposit and dissemination of scientific research documents, whether they are published or not. The documents may come from teaching and research institutions in France or abroad, or from public or private research centers.
L'archive ouverte pluridisciplinaire HAL, est destinée au dépôt et à la diffusion de documents scientifiques de niveau recherche, publiés ou non, émanant des établissements d'enseignement et de recherche français ou étrangers, des laboratoires publics ou privés. 


\title{
DIAGNOSIS OF AN UNCERTAIN STATIC SYSTEM
}

\author{
Olivier Adrot, Didier Maquin, José Ragot \\ Centre de Recherche en Automatique de Nancy - CNRS UPRESA 7039 \\ Institut National Polytechnique de Lorraine \\ 2, avenue de la Forêt de Haye - 54516 Vandœuvre-lès-Nancy Cedex, France \\ Phone: (33) 383595959 - Fax: (33) 383595644 \\ E-mail: \{Olivier.Adrot, Didier.Maquin, Jose.Ragot\}@ensem.inpl-nancy.fr
}

\begin{abstract}
This paper deals with an original fault detection and isolation method, allowing to take the structure and the range of model uncertainties into account. We focus on static and structured uncertain models, where each parameter uncertainty is described by a bounded variable. In order to de-couple residuals from unknown physical variables, a parity space approach is proposed, where the parity matrix depends on uncertain parameters. Because of this membership approach, called bounding approach, residuals represent a set of feasible behaviours and define therefore the normal operating domain of the studied physical system. To simplify its evaluation and work on a simple domain such as a parallelotope, residuals are linearised in the bounded variables and a reduction procedure is applied to decrease their complexity. Once the constraints defining this domain are determined, consistency tests for fault detection and isolation are built.
\end{abstract}

\section{Introduction}

Fault Detection and Isolation (F.D.I.) schemes generally use the concept of analytical redundancy based on a mathematical model of the studied system. A major drawback lies in the fact that a model only defines an approximate description of the physical system behaviour because of modelling errors occurring when it is linearised or some physical phenomena are neglected. Thus, to avoid confusing a modelling error with a fault, the inaccuracy of a model, represented by structured uncertain parameters, has to be taken into account.

This paper focuses on a F.D.I. method based on the bounding approach [8], [3], [7], taking the structure and the magnitude of each uncertain parameter into account. Thus, the values of some parameters are inaccessible and represented by a set of feasible values, which only the bounds are known. A parity space approach [5], suited to membership context, generates residuals allowing to confront the behaviour of the physical system with the reference model. Because of the inaccuracy of this model, residuals may be different from zero. They describe a set of feasible behaviours representing the normal operation field of the system. Its frontiers, built by using interval arithmetic [6] according to the ranges of uncertainties, naturally define the adaptive thresholds of the F.D. procedure.

Thus, this paper has the following structure. At first, we will limit ourselves to a class of static uncertain models and search an uncertain parity matrix allowing to generate residuals de-coupled from unknown physical variables. In the section 3, we stress on the problems caused by dependencies between bounded variables. A linearisation of the residuals in the uncertain parameters is proposed; in this way, the normal operation field is always a polytope [9], which can be easily evaluated. The fault detection and isolation procedures are respectively developed in section 4 and 5. At last, an example illustrates our method.

\section{Parity space approach}

\section{2.a. Presentation of the model}

We consider structured models, which allow to represent the lack of knowledge on a physical system by indicating which parameters are uncertain. These uncertainties are described by normalised bounded variables $\theta_{\mathrm{i}}$, which bounds are -1 and 1 . We are interested in the following class of static models composed at once of m measurement equations and $\mathrm{p}$ constraints on the $\mathrm{n}$ physical variables:

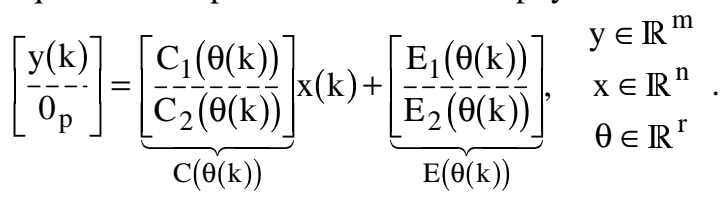

$\mathrm{y}$ is the sensor vector, while $\mathrm{x}$ and $\theta$ denote respectively the vectors containing physical variables and bounded variables. The distribution matrices $C_{i}$ and vectors $E_{i}$ are assumed to be linear in uncertain parameters. To remain general, a same uncertainty $\theta_{i}$ can affect several distribution matrices. In practice, the term $\mathrm{E}_{1}$ represents bounded sensory noises, whereas $\mathrm{C}_{1}$ describes the imperfection of the measurement system as sensor tolerances. $C_{2}$ and $E_{2}$ define the redundancy between physical variables.

To simplify the symbols, we will omit references to the fact that the previous relations depend on time, but notice that uncertain parameters fluctuate inside their bounds.

\section{2.b. Principle}

In order to generate residuals using the redundancy of the previous model (1), all the unknown physical variables $\mathrm{x}$ have to be eliminated. In this way, a parity space approach has been chosen, where an uncertain parity matrix $\mathrm{W}(\theta)$, such that $\mathrm{W}(\theta) \mathrm{C}(\theta)=0$, is searched. Notice that several works have already dealt with this problem in the case of a constant parity matrix for static systems [1] and dynamic ones [2], [3]. Unfortunately, a de-coupling with respect to physical (or state) variables is not always possible and depends on the structure of uncertainties (additive, multiplicative) and on the matrices of the state representation that they affect. That is the reason why another alternative is proposed in this paper.

The parity matrix $W(\theta)$ exists if the matrix $C(\theta)$ is not full row rank, which is the case in practice for a static system 
containing redundant information. Moreover, to evaluate the rank of $C(\theta)$, we will assume that it is invariant and independent of all the uncertainties. This assumption is natural because we are interested in parameter uncertainties, which cannot modify the structure of the model. It is improbable that an uncertainty changes the sign of a physical parameter or makes it equal to zero. Under these conditions, it can modify neither the observability nor the redundancy of the studied system. In fact, $C(\theta)$ has the same rank than its nominal part $\mathrm{C}_{0}=\mathrm{C}(0)$, which is a certain matrix. Then, to avoid the presence of redundant parity relations, $\mathrm{W}(\theta)$ has to be full row rank, and therefore, is composed of $s=(m+p)-\operatorname{rank}\left(C_{0}\right)$ independent rows.

Now, we will show that $\mathrm{W}(\theta)$ is a polynomial matrix in the uncertain parameters. The solution of the following problem $\mathrm{XA}=\mathrm{B}$, where $\mathrm{X}$ is unknown, is given by $\mathrm{X}=\mathrm{BA}^{+}+\mathrm{K}\left(\mathrm{I}-\mathrm{AA}^{+}\right)$where $\mathrm{K}, \mathrm{I}$ and $\mathrm{A}^{+}$are respectively an arbitrary matrix, the identity matrix and the pseusoinverse of the matrix $\mathrm{A}$ verifying: $\mathrm{AA}^{+} \mathrm{A}=\mathrm{A}$. By taking respectively $B=0, A=C(\theta)$, and assuming $C(\theta)$ is full column rank, the following expression is deduced:

$\mathrm{W}(\theta)=\mathrm{K}\left(\mathrm{I}_{\mathrm{m}+\mathrm{p}}-\mathrm{C}(\theta)\left(\mathrm{C}^{\mathrm{T}}(\theta) \mathrm{C}(\theta)\right)^{-1} \mathrm{C}^{\mathrm{T}}(\theta)\right)$,

where $\mathrm{K}$ is a matrix allowing to select all the independent rows. Since $\mathrm{K}$ is arbitrary, it can be replaced by $K \operatorname{det}\left(C^{T}(\theta) C(\theta)\right)$, where $\operatorname{det}(Z)$ is the determinant of $Z$. In this way, the simplest form of $\mathrm{W}(\theta)$ is a polynomial matrix in $\theta$. In case $\mathrm{C}(\theta)$ is not full column rank, it can always be broken down into $C(\theta)=C^{\prime}(\theta) C^{\prime \prime}(\theta)$ where matrices $C^{\prime}$ and $C$ " are both full column rank. Therefore, the problem is equivalent to find a matrix $\mathrm{W}(\theta)$ orthogonal to $C^{\prime}(\theta)$. In practice, we find the parity matrix using the symbolic computation software Mapple V.

Now, after multiplying the model (1) by $\mathrm{W}(\theta)$, the following expression of the residuals is deduced:

$$
\mathrm{R}(\theta)=\mathrm{W}(\theta)\left(\left[\begin{array}{c}
\mathrm{y} \\
\overline{0}_{\mathrm{p}}^{-}
\end{array}\right]-\mathrm{E}(\theta)\right)=0 \text {. }
$$

In the next section, we will show how to reformulate this system to determine the normal operation field.

\section{Linearisation and reduction procedures}

\section{3.a. Linearisation procedure}

At first, the notion of abstract space, denoted by $\mathscr{A}()$, is presented to tackle models containing uncertainties by bounding approach. If $\mathrm{Z}$ is a vector of bounded variables, in other words, if it is only known by the space to which it may belong, then this space is given as $\mathscr{A}(\mathrm{Z})$.

Let us assume that $\mathrm{Z}$ is an uncertain vector, which components depend on $\theta$. If no bounded variable affects several components at once, $\mathcal{A}(\mathrm{Z})$ leads to an axis-aligned orthotope. Nevertheless, if at least one bounded variable, called common variable, appears in several components $Z_{i}$ of $\mathrm{Z}$, the shape of $\mathcal{A}(\mathrm{Z})$ is bent because of dependencies between these $\mathrm{z}_{\mathrm{i}}[3]$. In this case, the abstract space $\mathcal{A}(\mathrm{Z})$ is included in its axis-aligned circumscribed orthotope, noted $\square, \mathcal{A}(\mathrm{Z})$, built with the help of interval arithmetic by assuming no variable is common (that is to say by treating independently each $\mathrm{z}_{\mathrm{i}}$ ). In practice, an orthotope is easier to compute. But, in case $\mathcal{A}(\mathrm{Z})$ represents the normal operation field of the studied system, the use of the domain $\square, \mathcal{A}(\mathrm{Z})$ instead of $\mathcal{A}(\mathrm{Z})$ decreases the precision of the F.D. procedure. An important part of the information is lost and the no-detection rate may become important.

If $Z(\theta)$ is non linear in some common variables $\theta_{i}$, the associated abstract space $\mathcal{A}(\mathrm{Z})$ is generally non linear and non convex, that is the reason why computing it remains difficult. The solution developed in [3] is based on the notion of paving. Thus, an overestimation of $\mathscr{A}(\mathrm{Z})$ is defined by the union of axis-aligned orthotopes obtained by the uniform subdivision of the hypercube $\mathscr{A}(\theta)$. An algorithm is proposed in order to limit time computing by searching the part of $\mathcal{A}(\mathrm{Z})$ useful to scan. Unfortunately, the precision of the method depends on the number of subdivisions, and unfortunately, greater it is, more important time consuming is.

By noticing that the expression (2) is non-linear in $\theta$, we deduced that $\mathscr{A}(\mathrm{R})$ is a complex domain. Under these conditions, this paper proposed an alternative, which is a compromise between precision and time consuming. The method consists in a linearisation of all the residuals in uncertain parameters, since, in this way, the approximate normal operation field becomes a convex polytope, which can be easily evaluated. By putting together the linear part of (2), the following expression is obtained:

$$
\mathrm{R}(\theta)=\underbrace{\mathrm{R}_{\mathrm{L}}(\theta)}_{\text {terms of order } \leq 1}+\underbrace{\mathrm{R}_{\mathrm{NL}}(\theta)}_{\text {terms of order } \geq 2},
$$

where the subscripts $\mathrm{L}$ and NL define respectively the linear and non-linear parts of the residual vector $\mathrm{R}$. Notice that both matrices $R_{(.)}$depend on measurements.

At first, for each component of $\mathrm{R}_{\mathrm{NL}}$, all identical monomials composed of bounded variables are put together. Then, every monomial is replaced by a new normalised and independent bounded variable. By noticing, with the help of interval arithmetic, that the product $\theta_{i} \theta_{j}$ belongs to the interval $[-1,1]$ if $\mathrm{i}$ and $\mathrm{j}$ are different, else $[0,1]$, the following properties are deduced:

if all powers intervening in the studied monomial are even, this one is equal to [0,1],

if at least one power is odd, it is equal to $[-1,1]$.

Therefore, in the latter case, a monomial as $m_{1}=\theta_{i}^{2} \theta_{j}, i \neq j$, for example, is straight replaced by a new normalised independent bounded variable $v_{1} \in[-1,1]$, whereas in the former case, a monomial as $\mathrm{m}_{2}=\theta_{\mathrm{i}}^{4} \theta_{\mathrm{j}}^{2}$ is described by a non centred interval: $v_{2} \in[0,1]$. In order to lose the minimum of information, a monomial is represented by the same bounded variable in all the residuals.

Thus, the equality (3) becomes :

$\mathrm{R}_{\mathrm{p}}(\theta, v)=\mathrm{R}_{\mathrm{L}}(\theta)+\mathrm{R}_{\mathrm{NL}}(\mathrm{v})$,

where $v$ is the bounded vector obtained during the linearization and the matrix $\mathrm{R}_{\mathrm{NL}}$ is linear in $\mathrm{v}$. 
This procedure leads to a loss of information since some dependencies are eliminated. For example, the relationship between both monomials $\mathrm{m}_{1}$ and $\mathrm{m}_{2}$ is not conserved because they are replaced by two independent variables. Therefore, the abstract space of $R_{p}(4)$ is necessary an overestimation of $\mathcal{A}(\mathrm{R})$ (3). In the former case, $\mathrm{R}_{\mathrm{p}}$ is a vector field $F(\theta, v)$ where all bounded variables $\theta_{i}$ and $v_{j}$ are independent. In the latter case, $\mathrm{R}$ corresponds to the same vector field $F\left(\theta, \theta_{M}\right)$, where $\theta_{M}$ contains every monomial of order greater than one. The dependencies between $\theta$ and $\theta_{M}$ generate some constraints, therefore, $\mathrm{F}\left(\theta, \theta_{\mathrm{M}}\right)$ is a particular case of $\mathrm{F}(\theta, v)$ and since $A\left(\theta_{\mathrm{M}}\right)=\mathscr{A}(\mathrm{v})$, each point of $\mathcal{A}\left(\mathrm{F}\left(\theta, \theta_{\mathrm{M}}\right)\right)$ is necessary a point of $\mathscr{A}(\mathrm{F}(\theta, \mathrm{v}))$.

By noticing that the interval $[0,1]$ is equal to $1 / 2-1 / 2[-1,1]$, it is always possible to reformulate (4) as follows:

$\mathrm{R}_{\mathrm{p}}(\theta, \mu)=\mathrm{R}_{\mathrm{L}}(\theta)+\mathrm{R}_{\mathrm{NL}}^{\prime}(\mu)$,

where $\mu$ is a vector of normalised bounded variables $\left(\|\mu\|_{\infty} \leq 1\right)$ and the matrix $\mathrm{R}_{\mathrm{NL}}^{\prime}$ is linear in $\mu$.

In conclusion, this procedure entertains a loss of precision, but anyway, for the kind of studied systems, the exact domain $\mathscr{A}(\mathrm{R})$ is often too much complicated to be used. Therefore, $\mathcal{A}(\mathrm{R})$ is overestimated by a polytope $\mathscr{A}\left(\mathrm{R}_{\mathrm{p}}\right)$. Nevertheless, the number of new bounded variables may be important and some of them can be put together and replaced by a unique term with adequate bounds without modifying the studied abstract space.

\section{3.b. Reduction procedure}

By putting together $\theta$ and $\mu$ in (5), this equality is deduced: $\mathrm{R}_{\mathrm{p}}(\mathrm{v})=\mathrm{M}(\mathrm{y}) \mathrm{v}+\mathrm{N}(\mathrm{y}), \quad v^{\mathrm{T}}=\left[\theta^{\mathrm{T}} \mu^{\mathrm{T}}\right],\|\mathrm{v}\|_{\infty} \leq 1$,

where the matrices $\mathrm{M}$ and $\mathrm{N}$ depend on measurements. The purpose of this procedure is the decreasing of the number of bounded variables in order to simplify the evaluation of the polytope $\mathcal{A}\left(\mathrm{R}_{\mathrm{p}}\right)$. Consider this example:

$R_{p}=\left[\begin{array}{c}v_{1}-2 v_{2} \\ 2 v_{1}-5 v_{2}\end{array}\right]$

By defining a new independent variable $v$ ' belonging to the interval $[-3,3]$, the previous expression can be written as:

$R_{p}^{\prime}=\left[\begin{array}{c}v^{\prime} \\ 2 v^{\prime}-v_{2}\end{array}\right]$.

Since $v^{\prime}$ is considered as independent from $v_{2}$, some information is lost and $\mathscr{A}\left(\mathrm{R}_{\mathrm{p}}\right)$ is included in $\mathscr{A}\left(\mathrm{R}_{\mathrm{p}}^{\prime}\right)$ [3]. The reduction procedure must not modify $\mathscr{A}\left(\mathrm{R}_{\mathrm{p}}\right)$, therefore every dependence has to be conserved. In fact, every bounded variable appearing in the form of a same linear combination in all components of $R_{p}$, can be put together and replaced by another one, independent of the remaining variables.

The alone difficulty is due to interval arithmetic for which the sum and the subtraction give the same interval:

$$
A\left(\sum \pm \alpha_{i} v_{i}\right)=\left(\sum\left|\alpha_{i}\right|\right)[-1,1], \quad \forall \alpha_{i} \in \mathbb{R} \text {. }
$$

Suppose that the symbol $\mathrm{m}_{\mathrm{ij}}$ denotes the $(\mathrm{i}, \mathrm{j})^{\text {th }}$ element of the certain matrix $M(6)$, whereas the set $\mathrm{I}_{\mathrm{NC}, \mathrm{i}}$ defines all the indexes of non common variables (that is to say the variables appearing at the most in one residual) intervening in the $i^{\text {th }}$ component of $R_{p}$. In a general manner, all $v_{j}$, $\mathrm{j} \in \mathrm{I}_{\mathrm{NC}, \mathrm{i}}$ can be collected and replaced by:

$\sum_{\mathrm{j} \in \mathrm{I}_{\mathrm{NC}, \mathrm{i}}}\left|\mathrm{m}_{\mathrm{ij}}\right| v^{\prime},\left|v^{\prime}\right| \leq 1$,

where $v$ ' is an independent normalised bounded variable.

For example, $\left[\begin{array}{c}v_{1}-v_{2} \\ v_{3}\end{array}\right]$ becomes $\left[\begin{array}{l}2 v^{\prime} \\ v_{3}\end{array}\right]$.

The same principle has to be applied on common variables. The procedure is a little more complicated because the sign with which the linear combination appears in the different rows of $\mathrm{M}(\mathrm{y}) \mathrm{v}$ has to be taken into account. Let us consider a combination, depending on some bounded variables $v_{j}$, which indexes define the set $\mathbf{J}$, and appearing in the rows of $\mathrm{M}(\mathrm{y}) \mathrm{v}$, which indexes correspond to the set $\mathrm{I}$. Let $\mathrm{i}_{0}$ be an arbitrary element of $I$ and $j_{1}$ the first element of $J$ taken as reference. A solution consists in determining the signs of every element $\mathrm{m}_{\mathrm{i}_{0} \mathrm{j}}, \mathrm{j} \in \mathrm{J}$. Then, for each row of index $\mathrm{i}$ belonging to $\mathrm{I}$, the combination is replaced by:

$\sum_{j \in J} \operatorname{sign}\left(m_{i_{0} j_{1}} m_{i_{0} j}\right) m_{i j} v^{\prime},\left|v^{\prime}\right| \leq 1$ with $\operatorname{sign}(x)=\left\{\begin{array}{c}1 \text { if } x>0 \\ -1 \text { if } x<0\end{array}\right.$.

For example, $\left[\begin{array}{ccc}1 & -2 & 2 \\ -2 & 4 & 1\end{array}\right]\left[\begin{array}{l}v_{1} \\ v_{2} \\ v_{3}\end{array}\right]$ becomes $\left[\begin{array}{cc}3 & 2 \\ -6 & 1\end{array}\right]\left[\begin{array}{c}v^{\prime} \\ v_{3}\end{array}\right]$

In this way, all summed terms have always the same sign in order to respect interval arithmetic. Thus, the alternation of the signs in a given column of $\mathrm{M}$ is taken into account. The multiplication of a whole column by -1 does not modify the abstract space $\mathcal{A}\left(\mathrm{R}_{\mathrm{p}}\right)$ since all the bounded variables are centred. Thus, the previous result is equivalent to $\left[\begin{array}{cc}-3 & 2 \\ 6 & 1\end{array}\right]\left[\begin{array}{c}v^{\prime} \\ v_{3}\end{array}\right]$ but differs from $\left[\begin{array}{ll}3 & 2 \\ 6 & 1\end{array}\right]\left[\begin{array}{c}v^{\prime} \\ v_{3}\end{array}\right]$

To do this work, a systematic numeric technique consists in testing two by two if the column vectors $\mathrm{m}_{\mathrm{j}_{1}}$ and $\mathrm{m}_{\mathrm{j}_{2}}$ of the matrix $\mathrm{M}$ are collinear. When the rank of the matrix $\left[\begin{array}{lll}\mathrm{m}_{\mathrm{j}_{1}} & \mathrm{~m}_{\mathrm{j}_{2}}\end{array}\right]$ is equal to $1, \mathrm{~m}_{\mathrm{j}_{1}}$ and $\mathrm{m}_{\mathrm{j}_{2}}$ are eliminated and replaced using the method explained above. The principle of the algorithm is detailed below:

(1) Determine the list L containing all the combinations of 2 elements among the number $\mathrm{q}$ of columns of $\mathrm{M}$.

(2) Take successively each element $l_{k}$ of $L$, until the rank of the matrix composed of the columns $\mathrm{m}_{\mathrm{j}_{1}}$ and $\mathrm{m}_{\mathrm{j}_{2}}$, which indexes $\left(\mathrm{j}_{1}, \mathrm{j}_{2}\right)$ are imposed by $l_{\mathrm{k}}$, is 1 . If there is no collinear column, go to the step (5).

(3) By assuming that the column of index $j_{1}$ is the reference, search one of its non-null elements, which will be noted $\mathrm{m}_{\mathrm{i}_{0} \mathrm{j}_{1}}$. A new column $\mathrm{m}$ is added to $\mathrm{M}$ as follows. If $\mathrm{m}_{\mathrm{i}_{0} \mathrm{j}_{1}}$ and $\mathrm{m}_{\mathrm{i}_{0} \mathrm{j}_{2}}$ (the latter is necessary nonnull too) have the same signs, then $m$ corresponds to the sum $\mathrm{m}_{\mathrm{j}_{1}}+\mathrm{m}_{\mathrm{j}_{2}}$, else the difference $\mathrm{m}_{\mathrm{j}_{1}}+\left(-\mathrm{m}_{\mathrm{j}_{2}}\right)$. At last, the columns $\mathrm{m}_{\mathrm{j}_{1}}$ and $\mathrm{m}_{\mathrm{j}_{2}}$ are suppressed.

(4) Do $q=q-1$; if $q$ is different from 1 , return to the step (1).

(5) Give the new matrix M. 
This procedure can be extended for symbolic expressions. In this case, the difficulty is caused by the measurements, which signs are unknown in advance and may change, whereas those of $\mathrm{m}_{\mathrm{i}_{0} \mathrm{j}_{1}}$ and $\mathrm{m}_{\mathrm{i}_{0} \mathrm{j}_{2}}$ have to be known. In fact, testing the collinearity of two columns is not more difficult than for the numeric case, since a symbolic expression common to both studied columns is simply searched. Moreover, the signs of $\mathrm{m}_{\mathrm{i}_{0} \mathrm{j}_{1}}$ and $\mathrm{m}_{\mathrm{i}_{0} \mathrm{j}_{2}}$ are not important, knowing whether they are different is enough.

Assume that an alone symbolic expression $f$ exists in at least one of both collinear columns. $f$ has to appear necessary in factor either in all elements of one column or in the elements of both columns having the same row indices. In the first case, since multiplying a column by \pm 1 does not modify the abstract space, $f$ can be replaced by its absolute value before putting together both columns:

For example, $\left[\begin{array}{cc}-1 & 2 \mathrm{f} \\ 2 & -4 \mathrm{f}\end{array}\right]\left[\begin{array}{l}v_{1} \\ v_{2}\end{array}\right]$ becomes $\left[\begin{array}{c}-1-2|\mathrm{f}| \\ 2+4|\mathrm{f}|\end{array}\right] v^{\prime}$.

In the second case, the absolute value of $\mathrm{f}$ cannot be generally used since modifying all signs of a column in the same manner is impossible. However, if the sign of $\mathrm{f}$ is unknown, it is trivial to notice that $\mathrm{f}$ and $-\mathrm{f}$ are opposite. In this way, if $\mathrm{m}_{\mathrm{i}_{0} \mathrm{j}_{1}}$ and $\mathrm{m}_{\mathrm{i}_{0} \mathrm{j}_{2}}$ have the same signs, both columns can be summed, else subtracted.

For example, $\left[\begin{array}{cc}-f & 2 f \\ 2 & -4\end{array}\right]\left[\begin{array}{l}v_{1} \\ v_{2}\end{array}\right]$ becomes $\left[\begin{array}{c}-3 f \\ 6\end{array}\right] v^{\prime}$ or $\left[\begin{array}{c}3 f \\ -6\end{array}\right] v^{\prime}$.

All other situations can be deduced from these both elementary cases. Therefore, the reduction procedure can be always applied to symbolic expressions after replacing every expression appearing in factor in one column and having an unknown sign by its absolute value.

\section{Fault detection method}

After using the reduction procedure on the relation (6), the following expression of residuals is obtained:

$\mathrm{R}_{\mathrm{p}}\left(\mathrm{v}^{\prime}\right)=\mathrm{M}^{\prime}(\mathrm{y}) \mathrm{v}^{\prime}+\mathrm{N}(\mathrm{y})=0, \quad\left\|v^{\prime}\right\|_{\infty} \leq 1, \mathrm{R}_{\mathrm{p}} \in \mathbb{R}^{\mathrm{s}}$

When no fault is present, a particular value $v_{0}$ ' of $v$ ', element of $\mathbb{R}^{\mathrm{q}}$, exists such that:

- the model is consistent with measurements, entertaining the nullity of the residual vector $\mathrm{R}_{\mathrm{p}}\left(\mathrm{v}_{0}\right.$ ')

- $\quad v_{0}$ ' is a feasible value in the sense that $v_{0}$ ' belongs to the hypercube $A\left(v^{\prime}\right)$.

The consistency test can be proceeded by searching $v_{0}$ ' at each time such that it belongs to $\mathscr{A}\left(v^{\prime}\right)$ and $\mathrm{R}_{\mathrm{p}}\left(v_{0}{ }^{\prime}\right)$ is equal to 0 . In fact, this test can be favourably replaced by the following one. The abstract space $\mathcal{A}\left(\mathrm{R}_{\mathrm{p}}\right)$ defines all the feasible values of the parity vector $R_{p}$, which are consistent with the chosen model according to $\mathscr{A}\left(\mathrm{v}^{\prime}\right)$. Therefore, $\mathcal{A}\left(\mathrm{R}_{\mathrm{p}}\right)$ represents the normal operation field of the physical system. A fault is detected if $R_{p}\left(v_{0}{ }^{\prime}\right)$, that is to say 0 , does not belong to $\mathscr{A}\left(\mathrm{R}_{\mathrm{p}}\right)$.

Notice that if the model is initially complete [4] (representative of every normal behaviour of the physical system), an inconsistency necessary guarantees the presence of a fault since the linearisation procedure leads to an overestimation $\mathcal{A}\left(\mathrm{R}_{\mathrm{p}}\right)$ (7) of $\mathcal{A}(\mathrm{R})$ (3). On the contrary, a consistency does not assure the absence of a fault, which may be masked by some uncertainties.

The construction of the polytope $\mathcal{A}\left(\mathrm{R}_{\mathrm{p}}\right)$ uses the algorithm presented in [7], which is easy to implement. More precisely, $\mathcal{A}\left(\mathrm{R}_{\mathrm{p}}\right)$ is a parallelotope centred in $\mathrm{N}(\mathrm{y})$, in other words a convex domain delimited by two by two parallel hyperplans (strip constraints). In fact, $\mathscr{A}\left(\mathrm{R}_{\mathrm{p}}\right)$ is the intersection of strip constraints, written in a general manner $\left\{\mathrm{R}_{\mathrm{p}} \in \mathbb{R}^{\mathrm{s}} / \mathrm{A}(\mathrm{y}) \mathrm{R}_{\mathrm{p}} \leq \mathrm{b}(\mathrm{y})\right\}$ [9]. This algorithm allows to generate the matrix $\mathrm{A}(\mathrm{y})$ and the vector $\mathrm{b}(\mathrm{y})$, which proceed from the constraints associated with the axis-aligned circumscribed orthotope $\square, \mathcal{A}\left(\mathrm{R}_{\mathrm{p}}\right)$ and from the elimination of common variables influencing the shape of $\mathscr{A}\left(\mathrm{R}_{\mathrm{p}}\right)$.

Therefore, testing whether the origin $\mathrm{O}$ belongs to $\mathscr{A}\left(\mathrm{R}_{\mathrm{p}}\right)$ is trivial by verifying whether the inequality $0 \leq \mathrm{b}(\mathrm{y})$ is true. However, the dichotomous nature of this test may appear somewhat poor according to all the information at our disposal. Instead of only testing whether $\mathrm{O}$ is inside $\mathscr{A}\left(\mathrm{R}_{\mathrm{p}}\right)$, it is possible to calculate the distance, as proposed in [7], separating $\mathrm{O}$ to the nearest border of the parallelotope. This result is then divided by the distance separating the centre $\mathrm{N}$ of $\mathscr{A}\left(\mathrm{R}_{\mathrm{p}}\right)$ and the same border. In a sense, this distance is normalised since the "size" of $A\left(\mathrm{R}_{\mathrm{p}}\right)$ is taken into account. At last, when the measurements are consistent, the obtained distance is multiplied by -1 . In this way, a distance of -1 means that the origin is merged with the centre $\mathrm{N}$, whereas the value 1 means that $\mathrm{O}$ is outside $\mathscr{A}\left(\mathrm{R}_{\mathrm{p}}\right)$ and as far from the border as $\mathrm{N}$ is from this border. Thus, the more this distance tends to zero, the more we find ourselves at the limit between consistency and inconsistency.

\section{Fault isolation method}

We will limit ourselves to the study of a single fault, which may appear on a sensor. Once a fault is detected, a F.I. procedure is applied to determine the faulty sensor. Since the objective is the use of the whole information at our disposal, it is natural to integrate strip constraints in this procedure. A table of signatures $S_{t}$ is created, which is not associated with residuals $R_{p}$ themselves, but with the inequality constraints defining the shape of $\mathscr{A}\left(\mathrm{R}_{\mathrm{p}}\right)$. In fact, there are always at least $\mathrm{s}$ strip constraints (these associated with each residual of $\mathrm{R}_{\mathrm{p}}$ and defining $\square, \mathcal{A}\left(\mathrm{R}_{\mathrm{p}}\right)$ ). As explained in the previous section, this number may increase with the presence of common variables since their elimination generates new strip constraints. In a general manner, $\mathcal{A}\left(\mathrm{R}_{\mathrm{p}}\right)$ leads always to more constraints than $\square, \mathcal{A}\left(\mathrm{R}_{\mathrm{p}}\right)$ and allows to isolate more different faults. Of course, it is possible to only use the former $\mathrm{s}$ strip constraints; but, in this case, a part of the information is lost since we work on $\square \mathscr{A}\left(\mathrm{R}_{\mathrm{p}}\right)$, which is less precise than $\mathscr{A}\left(\mathrm{R}_{\mathrm{p}}\right)$. Thus, a fault may be detected, but not isolated if the origin is outside $\mathcal{A}\left(\mathrm{R}_{\mathrm{p}}\right)$ but inside $\square, \mathscr{A}\left(\mathrm{R}_{\mathrm{p}}\right)$.

A fault is detected when the point $\mathrm{O}$ is outside $\mathcal{A}\left(\mathrm{R}_{\mathrm{p}}\right)$, in other words, when at least one constraint is not verified. In 
this way, the F.I. procedure consists in determining which strip constraints are not satisfied. By coding respectively with 0 and 1 the verified strip constraints and those, which are not satisfied, the signature $\mathrm{S}_{\mathrm{f}}$ of the fault is determined, which can be compared with the table of theoretic signatures $S_{t}$. Generally, the major difficulty to generate $S_{t}$ is due to the non-linear nature of $R_{p}(7)$ in measurements (this problem is caused by the elimination procedure of common variables). A bit 1 indicates that a strip constraint is theoretically sensitive to a given fault. But, this sensibility, which is usually deduced from a structural study of residuals linear in measurements, depends here on the numerical values of measurements. Consider the following static model leading to residuals $\mathrm{R}_{\mathrm{p}}$ :

$\left[\begin{array}{l}\mathrm{y}_{1} \\ \mathrm{y}_{2} \\ \mathrm{y}_{3}\end{array}\right]=\left[\begin{array}{l}1+0.1 \theta_{1} \\ 1+0.1 \theta_{2} \\ 1+0.1 \theta_{3}\end{array}\right] \mathrm{x}, \quad \mathrm{R}_{\mathrm{p}}=\left[\begin{array}{l}-\left(1+0.1 \theta_{2}\right) \mathrm{y}_{1}+\left(1+0.1 \theta_{1}\right) \mathrm{y}_{2} \\ -\left(1+0.1 \theta_{3}\right) \mathrm{y}_{1}+\left(1+0.1 \theta_{1}\right) \mathrm{y}_{3}\end{array}\right]$.

By eliminating analytically the common variable $\theta_{1}[8]$, we deduced this expression:

$\mathrm{r}_{3}=\mathrm{y}_{3} \mathrm{r}_{1}-\mathrm{y}_{2} \mathrm{r}_{2}=\mathrm{y}_{1}\left\{\left(1+0.1 \theta_{3}\right) \mathrm{y}_{2}-\left(1+0.1 \theta_{2}\right) \mathrm{y}_{3}\right\}$.

Since only a single fault is possible, when the first sensor is faulty, we can write:

$\mathrm{r}_{3}=\mathrm{y}_{1}\left(1+0.1 \theta_{3}\right)\left(1+0.1 \theta_{2}\right)(\mathrm{x}-\mathrm{x})=0$.

Notice that for determining the table of theoretic signatures, it is not necessary to calculate both constraints associated with a strip constraint by evaluating the lower and upper bounds of the residuals $r_{i}, i \in\{1,2,3\}$; the previous equalities are sufficient to indicate on which measurements each $r_{i}$ depends. For example, $\mathrm{r}_{3}$ structurally depends on $\mathrm{y}_{1}$, but is insensible to a fault on this measurement. In a more general manner, this difficulty is increased by the linearisation procedure, which modifies initial relations.

\begin{tabular}{|c|c|c|c|}
\cline { 2 - 4 } \multicolumn{1}{c|}{} & $\mathrm{r}_{1}$ & $\mathrm{r}_{2}$ & $\mathrm{r}_{3}$ \\
\hline $\mathrm{y}_{1}$ & 1 & 1 & $\mathbf{0}$ \\
\hline $\mathrm{y}_{2}$ & 1 & 0 & 1 \\
\hline $\mathrm{y}_{3}$ & 0 & 1 & 1 \\
\hline
\end{tabular}

Table 1. Table $\mathrm{S}_{\mathrm{t}}$ of theoretic signatures

In fact, theoretic signatures, based on a structural study of $\mathrm{R}_{\mathrm{p}}$, allow to obtain a basic table, which can be improved by an experimental study. The model, which "sensors" are successively affected by an important bias, is simulated and the obtained signatures allow to correct the theoretic ones. Thus, because of the non linear nature of $R_{p}$, some bits 1 of $\mathrm{S}_{\mathrm{t}}$ may be incorrect and thus replaced by 0 .

As for the F.D. procedure, a fault on the $\mathrm{i}^{\text {th }}$ sensor may be partially masked by uncertainties in the sense that its signature $S_{f}$ contains at least a bit 0 where its theoretic signature indicates a bit 1 . Notice that if the model is initially complete, the reverse is impossible since a constraint, which does not depend on the faulty measurement, is necessary satisfied. In order to compensate at best this problem, the signature $S_{\mathrm{f}}$ is corrected as follows. Let $p_{t}$ be the minimal number of bits 1 present in all the theoretic signatures. If the number $\mathrm{p}_{\mathrm{f}}$ of bits 1 in $\mathrm{S}_{\mathrm{f}}$ is lower than $\mathrm{p}_{\mathrm{t}}$, the constraint corresponding to the maximal negative normalised distance (as defined in the previous section) is searched. Against the F.D. procedure, the distances relative to all facets of $\mathcal{A}\left(\mathrm{R}_{\mathrm{p}}\right)$ have to be calculated. In this way, the objective is to find the constraint, which is satisfied with the smallest margin. Then, its representative bit 0 in $S_{\mathrm{f}}$ is replaced by 1 and the strip constraint associated with the found constraint is eliminated. This procedure is repeated until $\mathrm{p}_{\mathrm{f}}$ is equal to $\mathrm{p}_{\mathrm{t}}$. This technique limits the number of signatures $S_{f}$ which correspond to no theoretic ones and is particularly efficient when all theoretic signatures have the same number of bits 1. Notice that this correction does not guarantee the result: a fault may be confused with another one.

\section{Example}

To illustrate the previous developments, we use the example described in [7], that is the reason why we do not detail all equations. The uncertain model derives from the classic static model of the direct current machine:

$$
\left\{\begin{array}{c}
-\left(1+\rho_{1} \theta_{1}\right)[\mathrm{i}]+\left(1+\rho_{3} \theta_{3}\right) \frac{\gamma-1}{\gamma}[\omega]+\frac{[\mathrm{u}]}{\gamma}=0 \\
\left(1+\rho_{2} \theta_{2}\right)[\omega]-\left(1+\rho_{3} \theta_{3}\right)[\mathrm{i}]=0
\end{array}\right.
$$

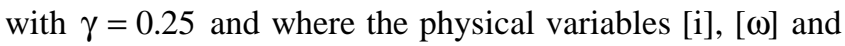
[u] corresponds to the current, the speed and the voltage supply, normalized according to their nominal values. The bounded variables $\theta_{\mathrm{i}}, \mathrm{i} \in\{1,2,3\}$ describe uncertainties respectively on the resistance, on the coefficient of viscous friction and on the electromagnetic constant. In the following, the symbol " $\sim$ " indicates that a variable is a measurement. Then, the power supply is assumed to be a perfectly known quantity, whereas, the measurements of current and speed are presumed to be imperfect:

$$
[\mathrm{u}(\mathrm{t})]=\tilde{\mathrm{u}}(\mathrm{t}), \quad\left\{\begin{array}{l}
\tilde{\mathrm{i}}=\left(1+\rho_{4} \theta_{4}\right)[\mathrm{i}]+\rho_{6} \theta_{6} \\
\tilde{\omega}=\left(1+\rho_{5} \theta_{5}\right)[\omega]+\rho_{7} \theta_{7}
\end{array}\right. \text {. }
$$

$\theta_{4}$ and $\theta_{5}$ define uncertainties of a multiplicative nature allowing to take into consideration the non-linearities of sensors. $\theta_{6}$ and $\theta_{7}$ are additive terms representing bounded sensor noises. All the bounded variables are normalized and the different coefficients $\rho_{i}$ define the range of the different uncertainties. The scalar $\rho_{1}$ equals 0.5 , which means that the resistance may vary by $\pm 50 \%$ around its nominal value. The value 0.2 is given to $\rho_{2}$ and $\rho_{3}$, while 0.05 represents the range of the four uncertainties $\rho_{\mathrm{i}}, \mathrm{i} \in\{4, \ldots 7\}$ of sensors.

We note $\theta_{i, j}$ the vector which contains, in increasing order of indices, the abstract variables $\theta_{k}, \mathrm{k} \in\{\mathrm{i}, \mathrm{i}+1, \ldots, \mathrm{j}\}$. By putting together the previous relations, we obtain the following static model of the direct current machine (1):

$\left[\begin{array}{c}\tilde{y} \\ 0\end{array}\right]=C\left(\theta_{1,5}\right)[y]+E\left(\theta_{6,7}\right)$

with $\tilde{\mathrm{y}}^{\mathrm{T}}=\left[\begin{array}{lll}\tilde{\mathrm{i}} & \tilde{\omega} & \tilde{\mathrm{u}}\end{array}\right]^{\mathrm{T}},[\mathrm{y}]^{\mathrm{T}}=[[\mathrm{i}][\omega][\mathrm{u}]]^{\mathrm{T}}$ and:

$$
\mathrm{C}\left(\theta_{1,5}\right)=\left[\begin{array}{ccc}
1+\rho_{4} \theta_{4} & 0 & 0 \\
0 & 1+\rho_{5} \theta_{5} & 0 \\
-0_{-} & 0 & -\frac{1}{\gamma}-\rho_{1} \\
-\left(1+\rho_{1} \theta_{1}\right) & \frac{1}{\gamma}\left(1+\rho_{3} \theta_{3}\right) & \frac{1}{\gamma} \\
-\left(1+\rho_{3} \theta_{3}\right) & 1+\rho_{2} \theta_{2} & 0
\end{array}\right], \mathrm{E}\left(\theta_{6,7}\right)=\left[\begin{array}{c}
\rho_{6} \theta_{6} \\
\rho_{7} \theta_{7} \\
0 \\
-0 \\
0 \\
0
\end{array}\right] \text {. }
$$


A parity matrix $\mathrm{W}\left(\theta_{1,5}\right)$ such that $\mathrm{W}\left(\theta_{1,5}\right) \mathrm{C}\left(\theta_{1,5}\right)=0$ is:

$$
\mathrm{W}\left(\theta_{1,5}\right)=\left[\begin{array}{cc}
\left(1+\rho_{3} v_{3}\right)\left(1+\rho_{5} v_{5}\right) & \gamma^{2}\left(1+\rho_{1} v_{1}\right)\left(1+\rho_{5} v_{5}\right) \\
-\left(1+\rho_{2} v_{2}\right)\left(1+\rho_{4} v_{4}\right) & \gamma(1-\gamma)\left(1+\rho_{3} v_{3}\right)\left(1+\rho_{4} v_{4}\right) \\
0 & -\gamma\left(1+\rho_{4} v_{4}\right)\left(1+\rho_{5} v_{5}\right) \\
0 & \gamma^{2}\left(1+\rho_{4} v_{4}\right)\left(1+\rho_{5} v_{5}\right) \\
\left(1+\rho_{4} v_{4}\right)\left(1+\rho_{5} v_{5}\right) & 0
\end{array}\right]^{\mathrm{T}}
$$

After multiplying (8) on the left-hand side by $\mathrm{W}\left(\theta_{1,5}\right)$, the linearisation procedure is applied. Thus, 15 new independent bounded variables are created in addition to $\theta_{1,7}$. Owing to the reduction procedure, the number of bounded variables is reduced to 7 . Using symbolic calculus, the expression of residuals (7) is obtained with:

$$
\begin{aligned}
& \mathrm{N}(\tilde{\mathrm{y}})=\left[\begin{array}{c}
\tilde{\mathrm{i}}-\tilde{\omega} \\
\gamma^{2} \tilde{\mathrm{i}}+\gamma(1-\gamma) \tilde{\omega}-\gamma \tilde{\mathrm{u}}
\end{array}\right], \\
& M^{\prime}(\tilde{y})=\left[\begin{array}{cr}
-\rho_{2}|\tilde{\omega}|-\rho_{36}-\rho_{27}-\rho_{356}-\rho_{247}-\rho_{35}|\tilde{\mathrm{i}}|-\rho_{24}|\tilde{\omega}| & \rho_{3} \tilde{\mathrm{i}} \\
0 & \gamma(1-\gamma) \rho_{3} \tilde{\omega}
\end{array}\right. \\
& \begin{array}{cccc}
\rho_{6}\left(1+\rho_{5}\right) & -\rho_{7}\left(1+\rho_{4}\right) & -\rho_{4} \tilde{\omega} & \rho_{5} \tilde{i} \\
\gamma^{2} \rho_{6}\left(1+\rho_{5}\right) & \gamma(1-\gamma) \rho_{7}\left(1+\rho_{4}\right) & -\gamma \rho_{4}((\gamma-1) \tilde{\omega}+\tilde{u}) & \gamma \rho_{5}(\gamma \tilde{i}-\tilde{u})
\end{array} \\
& 0 \\
& \left.\cdots \gamma^{2}\left(\rho_{1}|\tilde{\mathrm{i}}|+\rho_{16}+\rho_{156}+\rho_{15}|\tilde{\mathrm{i}}|\right)+\gamma(1-\gamma)\left(\rho_{37}+\rho_{347}+\rho_{34}|\tilde{\omega}|\right)+\gamma \rho_{45}|\tilde{\mathrm{u}}|\right]
\end{aligned}
$$

where the coefficient $\rho_{\mathrm{ijk}}$ defines the product $\rho_{\mathrm{i}} \rho_{\mathrm{j}} \rho_{\mathrm{k}}$.

Due to the presence of 5 common variables, the previous system leads to 7 strip constraints giving 7 residuals $r_{i}$, $i \in\{1, \ldots, 7\}$. At first, at each given moment, every strip constraints is evaluated and the distance separating the origin from the obtained parallelotope is assessed. A plus sign indicates that the origin $\mathrm{O}$ is situated outside $\mathcal{A}\left(\mathrm{R}_{\mathrm{p}}\right)$, and thus that the behavior of the system is not akin to the reference model (middle chart of figure 1). In the second phase, when an inconsistency is found, the F.I. procedure is used, where only the four strip constraints depending on a single measurement, are used. In this way, both theoretic signatures contain the same number of bits 1 .
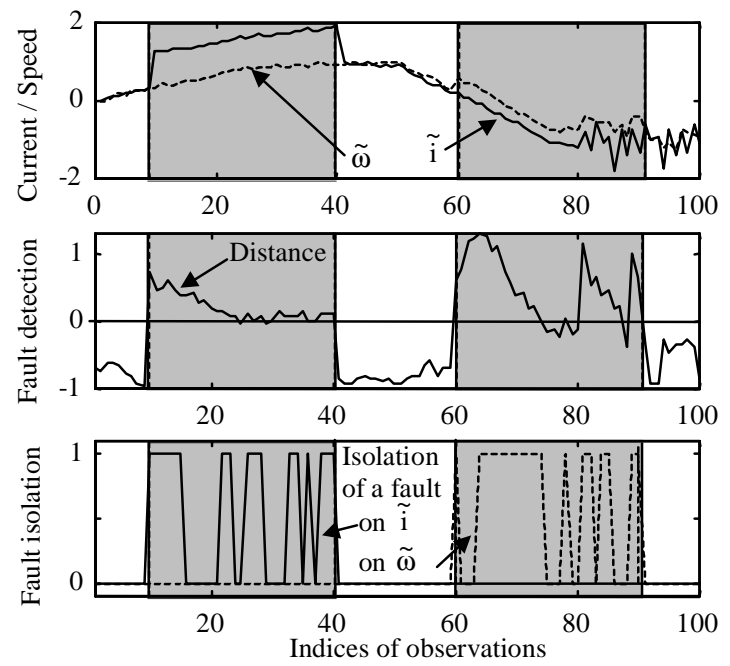

Figure 1. Results of the F.D.I. procedure

The system is simulated by adding two sensor biases and 100 observations are generated. For the observations, which index belongs to [10,40], a bias of magnitude 0.9 affects the current. For indices comprised between 60 and 90, a bias of magnitude 0.4 affects the speed. These faults may appear important, but the sum of uncertainty magnitudes rises to $\pm 110 \%$. As shown in the figure 1 , the results of this approach are guaranteed: an inconsistency necessarily reveals a behavioral anomaly. Both faults are well detected: $88 \%$ of the faulty measurements are detected. Moreover, only $11 \%$ of the faults are isolated, but this percentage rises to $70 \%$ when the signatures $S_{\mathrm{f}}$ of the fault are corrected. While $S_{f}$ does not contain two 1, the strip constraint satisfied with the smallest margin is searched and then $S_{f}$ is completed with another 1 . In the lower chart of the figure 1 , 1 indicates that the fault is isolated, whereas 0 is the sign of a signature, which corresponds to no theoretic signature.

\section{Conclusion}

This paper deals with a F.D.I. method suited to structured uncertain and static models. A parity space approach has been chosen to de-couple residuals from unknown physical variables. Then, consistency tests are based on the bounding approach, where parameter uncertainties are described by bounded variables. For allowing to evaluate the normal operating domain, residuals are linearised in the bounded variables and reduced to decrease their complexity. Once the constraints defining this domain are determined, consistency tests for the fault detection are trivial. The fault isolation uses the same principle and is improved by a correction based on the notion of distance. As extension, the use of a static form would allow to apply this method to uncertain dynamic systems.

\section{References}

[1] Adrot O., Ploix S., Ragot J., "Détection de défauts appliquée à des modèles statiques affectés par des incertitudes structurées“, GRETSI'99, (1999).

[2] Adrot O., Maquin D., Ragot J., "Fault detection with model parameter structured uncertainties“, ECC'99, Germany, 30 August-3 September, (1999).

[3] Adrot O., Maquin D., Ragot J., "Bounding approach to the fault detection of a class of uncertain dynamic systems", Safeprocess2000, Hungary, 14-16 June, (2000).

[4] Armengol J., Travé-Massuyès L., Vehi J., Lluis de la Rosa J., "A survey on interval model simulators and their properties related to fault detection", $14^{\text {th }}$ IFAC Beijing'99, pp 511-519, (1999)

[5] E.Y. Chow, A.S. Willsky. "Analytical Redundancy and the Design of Robust Failure Detection System“. IEEE Trans. Aut. Control, Vol. AC-29, No. 7, pp 603-614, (1984)

[6] Moore R.E., "Methods and applications of interval analysis", Studies in Applied Mathematics, (1979).

[7] Ploix S., Adrot O., Ragot J., "Bounding approach to the diagnosis of a class of uncertain static systems", Safeprocess2000, Hungary, 14-16 June, (2000).

[8] Ploix S., "Diagnostic des systèmes incertains: l'approche bornante", Ph.D. of the I.N.P.L., Nancy, France, (1998).

[9] Ziegler G.Z., "Lectures on polytopes", Graduate texts in Mathematics, Springer, (1998). 